\title{
Parameter Optimization of Linear Control System Based on Lyapunov Stability Theory
}

\author{
Shengguo Zhang ${ }^{1, ~ a, ~ S h u o ~ Z h a n g ~}{ }^{2, b}$, Guoheng Zhang ${ }^{1, c}$ and Wanchun Liü, d
}

${ }^{1}$ School of Electrical Engineering, Northwest University for Nationalities, Lanzhou, 730030, China

${ }^{2}$ School of Automation, Hangzhou Dianzi University, Hangzhou, 310018, China

azhangshengguo@tsinghua.org.cn, b407459957@qq.com, ${ }^{c} 123163154 @ q q . c o m$,

d1054828395@qq.com

Keywords: Lyapunov function, Optimal parameter, Lyapunov equation, Performance index.

Abstract: This paper aims at optimizing the system parameter of the control system with adjustable parameter. The optimization performance index is represented as a Lyapunov function which is decided by a symmetrical positive definite matrix and system's initial conditions. This symmetrical positive definite matrix, which is dependent upon the adjustable system parameter, is derived by solving Lyapunov equation so that system's asymptotical stability can be guaranteed. By doing so, the system parameter optimization problem is changed to be the performance index function extremum problem. Then by solving the partial derivative equation, the extreme point, i.e. the optimal system parameter is determined. Application example indicates that system's adjustable parameter is optimally selected in the sense that the control system is asymptotically stable as well as the control system performance index is the minimum.

\section{Introduction}

Some control systems, for instance motion control systems, their system parameters are not timevarying but adjustable, because systems' physical structure parameters such as mass, inertia, and rigidity etc. have multi alternatives [1]. These system parameters, on the one hand, provide the basis of physical structure design and on the other hand, also decide the systems' control performances. From the viewpoint of control, different system parameters might make the same control system acquire different relative stabilities and dynamic performances. So there is a system parameter optimization problem in the light of performance requirements for many control systems [2, 3].

In this paper, control system parameters are optimized based on Lyapunov stability theory [4, 5]. By Lyapunov equation, a symmetric positive definite matrix, which can guarantee system's asymptotical stability, is solved. This matrix is dependent upon optimized parameter. Then, defining system performance index function and this performance index function is also dependent upon optimized parameter. Last but not the least, the extremum of performance index function to the optimized parameter is solved. Thus, the optimal system parameter is derived. There still is an example to verify this parameter optimization.

\section{Parameter Optimization Method Based on Lyapunov Stability Theory}

A linear time-invariant (LTI) control system can be represented by state space model as:

$$
\left\{\begin{array}{l}
\boldsymbol{X}=\boldsymbol{A}(\gamma) x+\boldsymbol{B} u, \quad x(0)=x_{0} \\
y=\boldsymbol{C} x
\end{array}\right.
$$

where $x$ is state vector, $x(0)=x_{0}$ is initial state vector, $u$ is control input vector, and $y$ is output vector. $\boldsymbol{B}$ and $\boldsymbol{C}$ are input and output matrixes with appropriate dimensions, respectively. $\boldsymbol{A}(\gamma)$ is the system state matrix and $\gamma$ is the adjustable system parameter. There needs to find an optimal system parameter $\gamma$, so that the system is asymptotically stable and the system performance index function $J$ is the minimum, simultaneously: 


$$
J=\int_{0}^{\infty} x^{\mathrm{T}} \boldsymbol{W} x \mathrm{~d} t
$$

where $\boldsymbol{W}$ is a symmetrical positive definite weighting matrix. This is called a parameter optimization problem. By parameter optimization, it can not only make system asymptotically stable, but also make system achieve better performances such as shorter settle time, less overshoot etc.

Lyapunov Function and Lyapunov Equation. In accordance with Lyapunov stability theory, when the energy of an autonomous system has been decreasing with the increase of the time and at last reducing to be zero during system's freedom movement, this autonomous system is asymptotically stable. Under this consideration, a quadratic energy function $V(x)$ is introduced:

$$
V(x)=x^{\mathrm{T}} \boldsymbol{P} x,
$$

where $\boldsymbol{P}$ is an arbitrary symmetrical positive definite matrix. The function $V(x)$ is called Lyapunov function and its differential function $\&_{x}$ ) can be solved and denoted by:

$$
\mathscr{\&}(x)=\frac{\mathrm{d} V(x)}{\mathrm{d} t}=-x^{\mathrm{T}} \boldsymbol{Q} x
$$

where $\boldsymbol{Q}$ is another arbitrary symmetrical positive definite matrix.

There can be observed from Eq. 3 and Eq. 4, $V(x)$ is a non-negative function and $(x)$ is a non-positive function both about system state $x . V(x)$ represents the system energy and $\&_{x}$ ) represents the decreasing velocity of the system energy.

If there can find such symmetrical positive definite matrixes $\boldsymbol{P}$ and $\boldsymbol{Q}$, as well as they meet an algebraic equation, i.e. Lyapunov equation:

$$
\boldsymbol{A}^{\mathrm{T}} \boldsymbol{P}+\boldsymbol{P A}=-\boldsymbol{Q},
$$

where $\boldsymbol{A}$ is the state matrix of controlled system, and then, this controlled system is asymptotically stable in the sense of Lyapunov.

System Parameter Optimization. Because the controlled system is asymptotically stable, by solving the integration of Eq.4, there can be derived:

$$
\begin{aligned}
-\int_{0}^{\infty} x^{\mathrm{T}} \boldsymbol{Q} x & =\int_{0}^{\infty} \frac{\mathrm{d} V(x)}{\mathrm{d} t} \mathrm{~d} t \\
& =V(x(\infty))-V(x(0))=-V(x(0))=-x_{0}^{\mathrm{T}} \boldsymbol{P} x_{0},
\end{aligned}
$$

and if selecting symmetrical positive definite matrix $\boldsymbol{Q}=\boldsymbol{W}$, there gets:

$$
\int_{0}^{\infty} x^{\mathrm{T}} \boldsymbol{W} x=x_{0}^{\mathrm{T}} \boldsymbol{P} x_{0},
$$

where $\boldsymbol{P}$ is the symmetrical positive definite matrix solution of Lyapunov equation:

$$
\boldsymbol{A}^{\mathrm{T}}(\gamma) \boldsymbol{P}+\boldsymbol{P A}(\gamma)=-\boldsymbol{W} .
$$

Because $\boldsymbol{A}$ is dependent upon adjustable parameter $\gamma$, the solution of Eq. $8, \boldsymbol{P}$, is of course dependent upon $\gamma$, i.e. $\boldsymbol{P}=\boldsymbol{P}(\gamma)$. Then the performance index function that Eq. 2 represents is turned to be:

$$
J=\int_{0}^{\infty} x^{\mathrm{T}} \boldsymbol{W} x \mathrm{~d} t=x_{0}^{\mathrm{T}} \boldsymbol{P}(\gamma) x_{0} .
$$

Thus, the system parameter optimization problem is converted to be a performance index function minimization problem: 


$$
\min \left(J=x_{0}^{\mathrm{T}} \boldsymbol{P}(\gamma) x_{0}\right),
$$

where $\boldsymbol{P}(\gamma)$ meets Eq. 8. Once the initial condition $x_{0}$ is given, the optimal system parameter $\gamma$ can be solved by partial derivative equation:

$$
\frac{\partial J}{\partial \gamma}=0
$$

\section{Application Example}

Fig. 1 shows a negative unit feedback system which has the adjustable parameter $\gamma$. The system input is $r$ and system output is $y$. The deviation between input $r$ and output $y$ is $e$. For the purpose of decreasing the deviation between the input and the output, as well as decreasing the deviation varying rate in the dynamic process, the performance index is determined as:

$$
J=\int_{0}^{\infty}\left(e^{2}+\&\right) \mathrm{d} t
$$

The optimization task is to determine the optimal parameter $\gamma$, so that the performance index $J$ is the minimum under the conditions that the initial system state is zero and the system input is unit step signal $r=1(t)$.

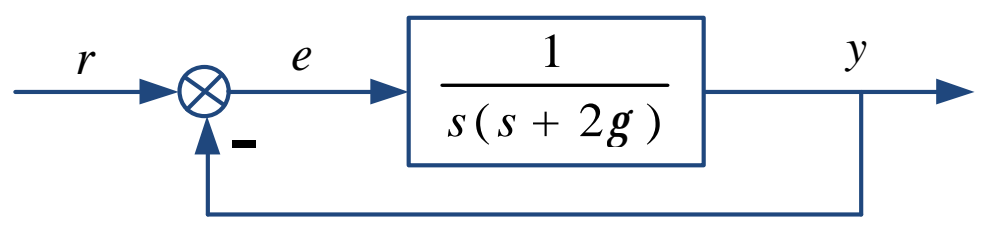

Fig. 1 The negative unit feedback system which has the adjustable parameter $\gamma$

From Fig.1, the closed-loop transfer function can be derived as:

$$
\Phi(s)=\frac{Y(s)}{R(s)}=\frac{1}{s^{2}+2 \gamma s+1} .
$$

Then using inverse Laplace transformation, the system differential eqation can be derived as:

$2 \gamma \propto+y=r$.

Because $e=r-y, r=1(t), \delta(0)=1$, and $\$(0)=0$, there gets another equation:

$2 \gamma \ll+e=0$,

Defining new state vector $x$ as:

$$
x=\left[\begin{array}{l}
x_{1} \\
x_{2}
\end{array}\right]=\left[\begin{array}{l}
e \\
e
\end{array}\right],
$$

there can get the closed-loop system state equation as:

$$
\mathcal{\&}=\left[\begin{array}{rc}
0 & 1 \\
-1 & -2 \gamma
\end{array}\right] x, x(0)=x_{0}=\left[\begin{array}{l}
x_{1}(0) \\
x_{2}(0)
\end{array}\right]=\left[\begin{array}{l}
1 \\
0
\end{array}\right] .
$$

Correspondingly, the performance index $J$ can be derived as: 


$$
\begin{aligned}
J & =\int_{0}^{\infty}\left(e^{2}+\&\right) \mathrm{d} t=\int_{0}^{\infty}\left(x_{1}^{2}+x_{2}^{2}\right) \mathrm{d} t=\int_{0}^{\infty}\left[\begin{array}{ll}
x_{1} & x_{2}
\end{array}\right]\left[\begin{array}{ll}
1 & 0 \\
0 & 1
\end{array}\right]\left[\begin{array}{l}
x_{1} \\
x_{2}
\end{array}\right] \mathrm{d} t \\
& =\int_{0}^{\infty} x^{\mathrm{T}} \boldsymbol{W} x \mathrm{~d} t,
\end{aligned}
$$

where:

$$
\boldsymbol{W}=\left[\begin{array}{ll}
1 & 0 \\
0 & 1
\end{array}\right],
$$

and then using Eq. 9, the performance index $J$ is represented as:

$$
J=x^{\mathrm{T}}(0) \boldsymbol{P}(\boldsymbol{\gamma}) x(0),
$$

where $\boldsymbol{P}(\gamma)$ is the matrix solution of Lyapunov equation:

$$
\boldsymbol{A}^{\mathrm{T}}(\gamma) \boldsymbol{P}(\gamma)+\boldsymbol{P}(\gamma) \boldsymbol{A}(\gamma)=-\boldsymbol{W}, \boldsymbol{A}(\gamma)=\left[\begin{array}{rr}
0 & 1 \\
-1 & -2 \gamma
\end{array}\right] .
$$

Solving Eq. 19, there gets:

$$
\boldsymbol{P}(\gamma)=\left[\begin{array}{cc}
\gamma+\frac{2}{\gamma} & \frac{1}{2} \\
\frac{1}{2} & \frac{1}{2 \gamma}
\end{array}\right] .
$$

So the performance index $J$ is derived as:

$$
J=x^{\mathrm{T}}(0) \boldsymbol{P}(\gamma) x(0)=\gamma+\frac{1}{2 \gamma},
$$

then solving the partial derivative equation:

$$
\frac{\partial J}{\partial \gamma}=1-\frac{1}{2 \gamma^{2}}=0
$$

there gets the optimal system parameter to be $\gamma=\sqrt{2} / 2$.

\section{Conclusions}

For a control system with adjustable parameter, its adjustable parameter can be optimized on the basis of Lyapunov stability theory. The system performance index is represented as a Lyapunov function which is dependent on a symmetrical positive definite matrix and the initial system states. This symmetrical positive definite matrix is solved by Lyapunov equation which can guarantee system's asymptotical stability. And this symmetric positive definite matrix is still dependent upon the adjustable system parameter because the system state matrix is dependent upon the adjustable parameter. Then the performance index function, which is dependent on the adjustable system parameter, is minimized by solving function extremum. The solved parameter is just the optimal system parameter which enables the system performance index minimization as well as guarantees system's asymptotical stability.

\section{Acknowledgements}

This work was financially supported by National Natural Science Foundation of China (51465053) and Fundamental Research Funds for the Central Universities (31920140082). 


\section{References}

[1] Zhongyu Gao, Control Engineering of Electromechanical Systems, third ed., Tsinghua University Press, Beijing 2011.

[2] Li Yu, Modern Control Theory, Tsinghua University Press, Beijing 2007.

[3] Siying Zhang, Liqun Gao, Modern Control Theory, Tsinghua University Press, Beijing 2006.

[4] A. Bacciotti and L. Rosier, Lyapunov Functions and Stability in Control Theory, Springer-Verlag 2005.

[5] M. Sassano and A. Astolfi, Dynamic Lyapunov Functions: Properties and Applications, 2012 American Control Conference, 2012, 2571-2576. 